\title{
Proyección de costos en Colombia de la atención inicial del síndrome coronario agudo
}

\author{
Julio César Castellanos Ramírez, MD ${ }^{1}$
}

\section{Resumen}

Se realizó un estudio retrospectivo de costo de la atención inicial de los casos de síndrome coronario agudo en el Hospital Universitario San Ignacio de la ciudad de Bogotá, en el periodo comprendido entre enero y octubre de 2010.

Objetivo: Realizar una estimación del costo médico promedio y total que significa para Colombia la primera atención de estos eventos agudos.

Métodos: Se estudiaron 213 pacientes que se confirmaron como SCA con un promedio de estancia de 7 días, con una máxima de 55 días y mínima de 1 día.

Resultados: Se encontró costo global promedio general \$ 7.781.230 pesos, medicamentos \$1.948.366 pesos y antiagregantes \$ 103.572. Tomando como referencia los casos esperados de SCA para un año según el ajuste por mortalidad se estima un costo médico anual proyectado esperado o proyectado para Colombia. (Tasa de cambio utilizada $\$ 1.850$ pesos por dólar) US\$228.886.152,00 y Bogotá US\$ 42.325.685.

Conclusión: El costo de la atención del SCA agudo en Colombia tiene una posición media en los referentes utilizados, no obstante lo presentado se estima que significa al año un costo médico importante de cerca de un cuarto de billón de dólares para el país y de más de cuarenta millones de dólares para Bogotá. Finalmente este trabajo sólo constituye un abordaje inicial que presenta datos que servirán de referencia a otras instituciones para elaborar sus propios estudios y hacer los seguimientos al respecto.

Palabras clave: síndrome coronario agudo, economía, terapia.

1 Especialista en Gerencia Hospitalaria - MEP. Director General Hospital Universitario San Ignacio. 
Title

Projected cost in Colombia of acute coronary syndrome initial care

\begin{abstract}
Was conducted a retrospective study of the cost of initial management of cases of acute coronary syndrome in the San Ignacio University Hospital of Bogota, in the period between January and October 2010.
\end{abstract}

Objective: Make an estimate of the average medical cost and total mean for Colombia's first acute care of these events.

Methods: We studied 213 patients who were confirmed as ACS with an average stay of 7 days, with a maximum of 55 days and at least 1 day.

Result: We found overall average total cost $\$ 7,781,230$ pesos, general drugs of $\$ 1,948,366$ pesos and $\$ 1,948,366 \$ 103,572$ pesos. Reference to the expected cases of ACS for a year as adjusted for mortality estimates a projected annual medical cost anticipated or planned for Colombia (exchange rate used $\$ 1,850$ pesos per dollar) US\$ $228,886,152.00$ and US $\$ 42,325,685$ for Bogota.

Conclusions: The cost of initial care of acute ACS care in Colombia is a middle position in the references used, notwithstanding that the material presented is estimated that every year means a major medical cost of nearly a quarter of a billion dollars for the country and more forty million dollars to Bogotá. Finally this paper only provides an initial approach that provides data to serve as reference for other institutions to develop their own studies and make follow-ups about it.

Key words: acute coronary syndrome, economics, therapy.

\section{Introducción}

Por las limitaciones en obtener de manera general la información de es- tos costos para todo el país e incluso dada la limitación que en el país se tiene para acceder a información confiable y de uso público costos de tratamiento por diagnóstico, en general y en específico para este diagnóstico, el presente trabajo busca de manera objetiva y sistemática presentar una información concisa que sirva a los interesados en tener datos que permitan hacer estudios más profundos o tener una base de comparación.

\section{Materiales y métodos}

Por la limitación de tiempo y recursos para realizar un estudio formal de economía de la salud que proyectara los costos de atención de síndrome en estudio, el autor aplicó la siguiente metodología:

1. Proyecto la incidencia del síndrome coronario agudo para Bogotá y Colombia utilizando artículos y referentes internacionales obtenidos de la literatura.

2. Realizó una revisión de los pacientes atendidos por diagnósticos relacionados con el síndrome coronario agudo en el Hospital Universitario San Ignacio, de enero a octubre de 2010.

3. Se revisaron todas y cada una de las historias para confirmar cuáles casos cumplían con los criterios del SCA. 
4. Se obtuvieron todas las facturas correspondientes a esas atenciones y se elaboró una base de datos que contenía la siguiente información:

a. Identificación del paciente.

b. Sexo.

c. Edad.

d. Fecha de ingreso y egreso.

e. Días de estancia.

f. Estado al egreso.

g. Estadía en la UCI.

h. Diagnóstico.

i. Si se practicó cirugía.

j. Si se practicó arteriografía coronaria.

k. Costo total de la atención del SCA teniendo en cuenta solamente lo correspondiente a la fase aguda, se excluyeron los costos de ingreso a procedimientos programados posteriores.

1. Costo discriminado de los medicamentos.

m. Costo discriminado de los antiagregantes utilizados, incluido el dipiridamol. Si se practicó estudio de medicina nuclear.

\section{Limitaciones del estudio}

Además de las limitaciones inherentes a los datos de referencia sobre incidencia esperada y el utilizar proyecciones poblacionales, así como tener pocos referentes en la literatura internacional, se anotan a continuación algunas de las que atañen a la institución.

Como se hizo únicamente en el Hospital San Ignacio, por sus características específicas de mercadeo y tarifarios es importante hacer las siguientes anotaciones que limitan que sus proyecciones de costo sólo aplican si la mayoría de las instituciones tienen un referente similar en el país:

1. Desde hace cinco años el Hospital ha aumentado sus tarifas buscando obtener sus excedentes mediante el valor de sus servicios médicos y hospitalarios y ha ido disminuyendo gradualmente el porcentaje de costo de manejo y administración de medicamentos e insumos.

2. La tarifa de insumos y medicamentos es la misma para todos los clientes independiente de la tarifa pactada de servicios.

3. No se hizo diferencia de régimen al cual pertenecía el paciente, por tanto en este estudio hay una mezcla 
de pacientes de régimen subsidiado, contributivo, medicina prepagada $\mathrm{y}$ pacientes internacionales.

4. A las tarifas actuales el hospital es sostenible y permite un excedente del 2,99\% sobre ingresos.

\section{Referente clínico}

Para este estudio, y clasificar los casos como síndrome coronario agudo se tomaron las definiciones de la Sociedad Colombiana de Cardiología y Cirugía Cardiovascular, así:

El término «síndrome coronario agudo» se usa para referirse a una constelación de síntomas clínicos compatibles con isquemia miocárdica aguda, incluyendo los diferentes subtipos de infarto agudo del miocardio así como la angina inestable. El espectro del síndrome coronario agudo comprende los siguientes diagnósticos:

1. Infarto agudo del miocardio: a. Con elevación del ST. b. Sin elevación del ST.

\section{Angina inestable[1].}

Los diagnósticos se confirmaron con los criterios anotados en la misma referencia:

En el contexto de síndrome coronario agudo, los criterios diagnósticos de infarto agudo del miocardio son: - Detección del aumento y descenso de biomarcadores cardíacos (preferiblemente troponina) con al menos un valor mayor al percentil $99 \%$ del límite superior normal. - Evidencia de isquemia miocárdica dada por uno de los siguientes parámetros: a. Síntomas de isquemia. $b$. Cambios en el electrocardiograma que indiquen nueva isquemia (variaciones recientes del ST o nиevo bloqueo de rama izquierda). c. Aparición de ondas $Q$ patológicas en el electrocardiograma. d. Evidencia por imágenes de pérdida reciente de miocardio viable o una nueva anomalía en el movimiento de la pared miocárdica[2].

\section{Resultados}

Tomando la referencia del Instituto Nacional de Sangre, Corazón y Pulmón de los Estados Unidos (NHLBI), que reporta la incidencia anual de IAM de 565.000 nuevos eventos y 300.000 ataques recurrentes cada año[3], y aplicando esta misma proyección de incidencia con base en la población proyectada por el DANE para Bogotá y Colombia se esperarían al año 82.293 casos para el país y 13.462 para Bogotá[4].

Tomado los referentes de mortalidad[5] para hacer un ajuste se esperarían al año 61.512 casos para el país y 10.063 para Bogotá. 
Tomando el estudio Corpus Christi realizado entre 1988 y 1992[6]. Que establece el riesgo de incidencia y tomando los datos para población de origen hispano que refiere para los grupos de edad entre 25 y 75 años, la siguiente incidencia para mujeres 354 y para hombres 486 , al proyectarlo para Colombia se esperaría al año 95.479 casos para el país y 17.027 para Bogotá.

Teniendo en cuenta que los hábitos y factores de riesgo de la población colombiana difieren en general de los de la población hispana residente en Estados Unidos y que las ci- fras de mortalidad tienen mejor soporte de datos, para las estimaciones finales se tendrá en cuenta el número de casos ajustados por la mortalidad.

En el Hospital Universitario San Ignacio en el período estudiado se encontraron 385 pacientes cuyos diagnósticos estaban relacionados y de los cuales 213 finalmente se confirmaron como SCA con un promedio de estancia de 7 días, con una máxima de 55 días y mínima de 1 día.

La distribución, por estado al egreso, se muestra en la siguiente tabla:

Tabla 1. Estado de los pacientes al egreso

\begin{tabular}{lcccr}
\hline & Remitido & $\begin{array}{c}\text { Fallecido antes } \\
\text { de } \mathbf{4 8} \text { horas }\end{array}$ & $\begin{array}{c}\text { Fallecido después } \\
\text { de } \mathbf{4 8} \text { horas }\end{array}$ & De alta \\
\hline Número & 12 & 5 & 4 & 192 \\
Porcentaje & 6 & 2 & 2 & 90 \\
\hline
\end{tabular}

La distribución por tipo de intervención, teniendo en cuenta si fue médica o quirúrgica y la práctica de dos estudios especiales arteriografía coronaria y medicina nuclear, fue:

Tabla 2. Tipo de intervención

\begin{tabular}{lcccc}
\hline Quirúrgica & $\begin{array}{c}\text { Arteriografía } \\
\text { coronaria }\end{array}$ & $\begin{array}{c}\text { Manejo } \\
\text { médico }\end{array}$ & $\begin{array}{c}\text { Medicina } \\
\text { nuclear }\end{array}$ \\
\hline Número & 18 & 104 & 195 & 32 \\
Porcentaje & 8 & 49 & 92 & 15 \\
\hline
\end{tabular}


En cuanto al uso de antiagregantes, la distribución fue la siguiente: se usó Clopidrogel en 179 casos (84\%), ácido acetilsalicílico (ASA) en 194 casos (91\%) y Tirofiban en 5 casos $(2 \%)$.

Se analizó el costo en los 213 casos, encontrando los siguientes resultados:

Costo global promedio general $\$ 7.781 .230$ pesos, medicamentos $\$ 1.948 .366$ pesos y antiagregantes $\$ 103.572$, sin tener en cuenta los pacientes a los cuales se les suministró Tirofiban, el costo global promedio general que así: \$ 7.717.464 pesos, medicamentos $\$ 1.934 .414$ pesos $y$ antiagregante $\$ 62.822$ pesos.
Con relación a la proporción de precio entre los antiagregantes se estableció como unidad de referencia el costo de una presentación farmacéutica de la aspirina, encontrando que para la facturación del Hospital San Ignacio la tarifa del Clopidrogel equivale a 70 ASA y el Tirofiban a 14.185 ASA.

Con respecto al promedio global de costo de cada caso con relación al estado de salida del paciente y si hubo intervención quirúrgica, el resultado se presenta en la siguiente tabla en la cual además de establece una relación con el costo global promedio general, se excluyen los pacientes quirúrgicos del cálculo para costo por estado al egreso.

Tabla 3. Costo promedio por estado de los pacientes al egreso y si hubo cirugía

\begin{tabular}{|c|c|c|c|c|c|}
\hline & Cirugía & $\begin{array}{c}\text { Fallecido } \\
\text { antes de } \\
48 \text { horas }\end{array}$ & $\begin{array}{l}\text { Fallecido } \\
\text { después de } \\
48 \text { horas }\end{array}$ & De alta & Remitido \\
\hline $\begin{array}{l}\text { Costo } \\
\text { promedio }\end{array}$ & $\$ 27.143 .049,00$ & $\$ 6.631 .544,00$ & $\$ 38.063 .622,00$ & $\$ 5.606 .735,00$ & $\$ 6.063 .052,00$ \\
\hline $\begin{array}{l}\text { Relación } \\
\text { costo } \\
\text { global }\end{array}$ & 3,5 & 0,9 & 4,9 & 0,9 & 0,8 \\
\hline $\begin{array}{l}\text { Promedio } \\
\text { general }\end{array}$ & & & & & \\
\hline $\begin{array}{l}\text { Número } \\
\text { de casos }\end{array}$ & 18 & 5 & 2 & 176 & 12 \\
\hline
\end{tabular}


En cuanto a referencia internacional la literatura revisada, en la cual la moneda local se convirtió en dólares americanos a la tasa de cambio vigente el 22 de noviembre de 2010, mostró la siguiente información:

Tabla 4. Costo del SCA en otros países

\begin{tabular}{lr}
\hline País & Costo \\
\hline Tailandia[7] & $\$ 1.595,34$ \\
USA[8] & $\$ 8.114,00$ \\
Australia[9] & $\$ 74.740,00$ \\
\hline
\end{tabular}

Tomando como referencia los casos esperados de SCA para un año según el ajuste por mortalidad se estima un costo médico anual proyectado esperado o proyectado para Colombia (tasa de cambio utilizada $\$ 1.850$ pesos por dólar) US\$228.886.152,00 y Bogotá US\$ 42.325.685.

El costo por caso comparando países fue:

Tabla 5. Costo del SCA en Colombia y otros países

\begin{tabular}{lr}
\hline País & Costo \\
\hline Tailandia & $\$ 1.595,34$ \\
Colombia & $\$ 4.206,00$ \\
USA & $\$ 8.114,00$ \\
Australia & $\$ 74.740,00$ \\
\hline
\end{tabular}

\section{Discusión de resultados}

Los resultados presentados evidencian una relación de costo promedio aceptable con relación al estado del paciente y el tipo de intervención, el uso de antiagregantes nuevos de mayor costo no hace cambiar significativamente el costo promedio del caso y de los medicamentos más si el costo medio atribuible a este tipo de medicamentos.

Aunque el número de pacientes fallecidos después de 48 horas fue muy bajo 2, llama la atención el costo medio de 4.9 veces con respecto al general, revisando con detalle los dos casos eso se debió a que uno de los pacientes tuvo una estancia prolongada en la Unidad de Cuidado Intensivo, por complicaciones inherentes a su condición clínica.

Los datos de otros países son muy similares en el costo atribuible al evento agudo en los estudios de Tailandia y Estados Unidos, no así el caso de Australia, para que esta comparación, que se incluyó a título de referencia tenga un mayor valor comparativo habría que estudiar el costo medio estándar de al menos la cama hospitalaria y la hora de especialista en cardiología.

\section{Conclusiones}

Por las consideraciones antes expuestas este trabajo sólo constituye un abordaje inicial que presenta datos que servirán de referencia a otras instituciones para elaborar sus propios estudios y hacer los seguimientos al respecto. 
El costo de la atención del SCA agudo en Colombia tiene una posición media en los referentes utilizados, no obstante lo presentado se estima que significa al año un costo médico importante de cerca de un cuarto de billón de dólares para el país y de más de cuarenta millones de dólares para Bogotá.

Se requieren sobre el tema estudios de fármaco-economía que tengan en cuenta los árboles de desenlaces y la evaluación de nuevas o ya existentes tecnologías para aportar a los protocolos y guías existentes en el país elementos que permitan tener además del mejor desenlace clínico posible para el paciente una protección adecuada de los recursos del Sistema General de Seguridad Social.

\section{Bibliografía}

1. Mendoza $B$, Fernán del C, Isaza R, D, Beltrán P, R et al. Guías colombianas de cardiología. Síndrome coronario agudo con elevación del ST. Revista Colombiana de Cardiología. 2010; 17,S.3:134.

2. Mendoza B. Fernán del C, Isaza R, D, Beltrán P, R et al. Guías colombianas de cardiología. Síndrome coronario agudo con elevación del ST. Revista Colombiana de Cardiología. 2010; 17,S.3:134.

3. Cassiani M, CA. Cabrera G, A. Sindromes coronarios agudos: epidemiología y diagnóstico. Salud Uninorte. Barranquilla (Col.) 2009;25(1):118-34.
4. DANE. Proyecciones de población. Colombia. Proyecciones de población departamentales y municipales por área 2005 - 2020. Fecha de consulta: 11 de noviembre de 2010. Disponible en: http://www.dane.gov.co/files/investigaciones/poblacion/proyepobla06_20/ ProyeccionMunicipios2005_2020.xls

5. Cassiani M, CA. Cabrera G, A. Síndromes coronarios agudos: epidemiología y diagnóstico. Salud Uninorte. Barranquilla (Col.) 2009;25(1):118-34.

6. Goff DC, Milton Z, Nichaman, Wenyaw Chan et al. Greater Incidence of Hospitalized Myocardial Infarction Among Mexican Americans Than NonHispanic Whites. The Corpus Christi Heart Project, 1988-1992. Circulation. 1997;95:1433-40. Fecha de consulta: 19 de noviembre de 2010. Disponible en: http://circ.ahajournals.org/cgi/ content/full/95/6/1433

7. Moleerergpoom W, Kanjanavanit R, Jintapakorn W, Sritara P et al. Costs of Payment in Thai Acute Coronary Syndrome Patients. J Med Assoc Thai. 2007;90(Suppl. 1):21-30.

8. Gregory A. Nichols, Timothy J. Bell, MHA; Kathryn L. Pedula, MS et al. Medical Care Costs Among Patients With Established Cardiovascular Disease. Am J Manag Care. 2010; 16(3):e86- e93.

9. Access economics: The economic costs of heart attack and chest pain (Acute Coronary Syndrome), Canberra, Eli Lily, 2009. Fecha de consulta: 21 de noviembre de 2010. Disponible en: http://www.bakeridi.edu.au/Assets/ Files/FullReport $\% 20 \% 20$ the $\%$ 20economic $\% 20$ costs $\% 20$ of $\%$ 20heart $\% 20$ attack\%20and\%20chest \%20pain $\% 20$ (emilable.pdf. 